\title{
Die Rolle der Hormone in der Behandlung der Keratokonjunktivitis sicca
}

\author{
G. Schild
}

Ein immer größer werdender Anteil an Patienten in der ophthalmologischen Ordination klagt über zu trockene Augen, eine Keratokonjunktivitis sicca. Ursachen für die Veränderungen des Tränenfilms sind sowohl in exogenen als auch endogenen Faktoren $\mathrm{zu}$ suchen, wobei Veränderungen im Hormonhaushalt die größte Gruppe der endogenen Ursachen darstellen.

Da der weitaus größte Patientenanteil mit Trockenen Augen Frauen in der Menopause sind, waren zunächst die weiblichen Hormone das Ziel intensiver Forschung. Es hat sich jedoch gezeigt, dass die Rolle der Hormone am Auge vielschichtig und sehr komplex ist. Neben Östrogen und Progesteron spielen auch Veränderungen im Androgenhaushalt eine wichtige Rolle in der Entwicklung einer Keratokonjunktivitis sicca. Davon betroffen sind sowohl Frauen als auch Männer! Mit dem Nachweis hormonspezifischer Rezeptoren am äußeren Auge ist auch eine morphologische Grundlage für die Wirksamkeit von Hormonen am Auge gegeben.

Auch hormonelle Stoffwechselerkrankungen wie ein Diabetes mellitus und Schilddrüsenerkrankungen haben einen Einfluss auf die Entstehung der Keratokonjunktivitis sicca. Ein Nachweis von Insulin- oder Schilddrüsenhormonrezeptoren ist nach unserem Wissen bisher jedoch noch nicht gelungen, wenngleich es immunhistologisch indirekt Hinweise auf dessen Vorhandensein gibt.

Die Therapie der hormonell bedingten Keratokonjunktivitis sicca erfordert die interdisziplinäre Zusammenarbeit zwischen Ophthalmologen und Stoffwechselspezialisten. Die systemisch von den Gynäkologen angewendete Hormonersatztherapie scheint am Auge, so berichten die neuesten Studien, einen negativen Einfluss im Sinne einer Verschlechterung der Symptome zu bewirken. Auch lokal angewendete Salben führen oft nur zu einer geringen Linderung der Beschwerden. Die sehr geringe Rezeptorendichte könnte eine der Ursachen dafür sein.

In den meisten Fällen bleibt die Therapie daher auf den Ersatz der fehlenden Tränenflüssigkeitskomponenten beschränkt, wenngleich eine Therapie mit einem topisch applizierten Östrogen nie unversucht bleiben soll.

\section{Therapiekonzepte für das Trockene Auge (Übersicht)}

\author{
J. Nepp \\ Augenklinik der Medizinischen Universität Wien, Österreich (Vorstand: Prof. Dr. U. Schmidt-Erfurth)
}

Zusammenfassung. Die Therapie des Trockenen Auges wird empirisch mit verschiedenen Substanzen unternommen, die unterschiedlich gut ansprechen.

Wegen der unterschiedlichen Wirkung, aber auch der Weiterentwicklung des Wissens rund um das Trockene Auge wurden Therapiekonzepte vorgestellt, welche hier vorgestellt werden sollen.

1) Historische Mittel

2) Therapien bezogen auf die gestörte Phase des Tränenfilms

3) Ätiologiebezogene Therapie

4) Therapie entsprechend des Schweregrads

5) Operative Therapien

6) Sonstige empirische Therapien

7) Zukünftige Konzepte (Physikalische Differenzierung, Weiterentwicklung des Wissens um Hormone, Immunologie und Neuroimmunologie, sowie dynamische Kon- zepte aus der Tränenfilmphysiologie - vor allem nachts und Psychologie)

$\mathrm{Zu}$ beachten sind aber auch Schäden durch Konservierungsmittel, Kontaktlinsen, und subjektive Beschwerden.

All diese Konzepte sollen aber nicht vortäuschen, dass das ideale Therapiekonzept schon entdeckt wurde, und dass zur Zeit ein Optimum nur durch die individuelle Behandlung möglich ist.

Schlüsselwörter: Therapiekonzepte, trockene Augen, SiccaScore.

\section{Einleitung}

Das Trockene Auge stellt hinsichtlich der Diskrepanz der Beschwerden und der oft nur geringen Befunde oft ein Problem dar, vor allem da es darum geht, einerseits Komplikationen seitens der Hornhaut, wie tiefe Entzündungen, Substanz- 